\title{
Sequential Activation of Metabolic Pathways: a Dynamic Optimization Approach
}

\author{
Diego A. Oyarzún ${ }^{\mathrm{a}, *}$, Brian P. Ingalls ${ }^{\mathrm{b}}$, Richard H. Middleton ${ }^{\mathrm{a}}$, \\ Dimitrios Kalamatianos $^{\mathrm{a}}$ \\ ${ }^{a}$ Hamilton Institute, National University of Ireland Maynooth, Maynooth, Co. Kildare, \\ Ireland \\ ${ }^{b}$ Department of Applied Mathematics, University of Waterloo, Ontario, Canada, N2L $3 G 1$
}

Received: 22 September 2008 / Accepted: 15 April 2009 / Published online: 2 May 2009

(C) The Author(s) 2009. This article is published with open access at Springerlink.com

\begin{abstract}
The regulation of cellular metabolism facilitates robust cellular operation in the face of changing external conditions. The cellular response to this varying environment may include the activation or inactivation of appropriate metabolic pathways. Experimental and numerical observations of sequential timing in pathway activation have been reported in the literature. It has been argued that such patterns can be rationalized by means of an underlying optimal metabolic design. In this paper we pose a dynamic optimization problem that accounts for time-resource minimization in pathway activation under constrained total enzyme abundance. The optimized variables are time-dependent enzyme concentrations that drive the pathway to a steady state characterized by a prescribed metabolic flux. The problem formulation addresses unbranched pathways with irreversible kinetics. Neither specific reaction kinetics nor fixed pathway length are assumed.

In the optimal solution, each enzyme follows a switching profile between zero and maximum concentration, following a temporal sequence that matches the pathway topology. This result provides an analytic justification of the sequential activation previously described in the literature. In contrast with the existent numerical approaches, the activation sequence is proven to be optimal for a generic class of monomolecular kinetics. This class includes, but is not limited to, Mass Action, Michaelis-Menten, Hill, and some Power-law models. This suggests that sequential enzyme expression may be a common feature of metabolic regulation, as it is a robust property of optimal pathway activation.
\end{abstract}

Keywords Metabolic dynamics · Metabolic regulation · Dynamic optimization

\footnotetext{
*Corresponding author.

E-mail addresses: diego.oyarzun@nuim.ie (Diego A. Oyarzún), bingalls@math.uwaterloo.ca (Brian P. Ingalls), richard.middleton@nuim.ie (Richard H. Middleton), dimitris.kalamatianos@nuim.ie (Dimitrios Kalamatianos).
} 


\section{Introduction}

The behavior of metabolic pathways depends on the network structure and the kinetics of the enzymes catalyzing the individual reactions (Savageau, 1976; Heinrich and Schuster, 1996). These systems exhibit diverse dynamic behaviors in terms of stability, steady state and transient response. Most cellular processes rely on the appropriate operation of some set of pathways and so metabolic behavior underpins functional requirements for cellular operation. The existence of alternative metabolic designs for the same function (Savageau, 1985) together with the major role that metabolic dynamics play in cell fitness indicate that they have been optimized through evolutionary processes (Heinrich et al., 1991; Cornish-Bowden, 2004b).

The method of Mathematically Controlled Comparisons (MMC) (Savageau, 1976, 1985; Alves and Savageau, 2000) allows the comparison of alternative metabolic designs with respect to specific quantitative criteria. Among other applications, MMC has been effective in the study of optimal regulatory structures in metabolic pathways (Savageau, 1974, 1975). Another optimization technique that has been successfully applied to metabolic networks is Flux Balance Analysis (FBA) (Varma and Palsson, 1994), whereby the reaction fluxes of a stoichiometric model are chosen to optimize a linear objective function. FBA has provided useful predictions of metabolic responses in a number of different organisms under diverse conditions, e.g., Ibarra et al. (2002). Such predictions depend on the choice of an appropriate objective function, the selection of which is a subject of active research (Schuetz et al., 2007; Nielsen, 2007; Schuster et al., 2008). On the other hand, when reaction kinetics are available, a number of different optimization problems have been considered, e.g. flux optimization (Heinrich et al., 1991; Heinrich and Klipp, 1996; Holzhütter, 2004), minimization of total enzyme concentration (Klipp and Heinrich, 1999) and maximization of growth rate (Bilu et al., 2006). Since no single optimality criterion captures all relevant objectives, multicriteria optimization has also been proposed as a way of taking into account different metabolic objectives within a single framework (Vera et al., 2003).

Each of the aforementioned studies addresses the network behavior under static enzyme concentrations. However, the temporal distribution of enzymatic activity affects pathway behavior and metabolic responses are modulated by the timing of enzyme expression. Zaslaver et al. observed well defined temporal patterns in gene expression data in amino acid biosynthetic pathways of $E$. coli under extracellular medium shift (Zaslaver et al., 2004; Campbell, 2004). A sequential or "just-in-time" pattern in enzyme expression was found in the Serine, Methionine and Arginine pathways. Additional experimental evidence revealing temporal modulation in the Lysine pathway has been recently reported (Ou et al., 2008). These experiments provide metabolic instances of the generally accepted fact that specific temporal patterns in gene expression appear in the operation of a range of cellular functions, including complex molecular assemblies (Kalir et al., 2001) and organism development (Leng and Müller, 2006).

Rationalizing such temporal patterns by means of optimization principles requires ideas from dynamic optimization theory. To date, there have been relatively few studies on dynamic optimization of metabolic pathways (as observed in Torres and Voit, 2002, p. 165). In Varner and Ramkrishna (1999), the authors develop a theoretical framework where cells are regarded as optimal resource allocators following cybernetic principles, while extensions of the FBA principle to include dynamic behavior have 
been reported in a number of works (van Riel et al., 2000; Mahadevan et al., 2002; Uygun et al., 2006).

Dynamic enzyme optimization for the activation of biosynthetic pathways has been considered recently in Klipp et al. (2002) and Zaslaver et al. (2004). Klipp and coworkers obtained enzyme profiles that minimize the transition time (Llorens et al., 1999; Torres, 1994) of a thermodynamically closed unbranched pathway with Mass Action kinetics. The problem was posed under a constraint on the total enzyme abundance, which reflects the fact that cells have limited biosynthetic capability. Numerical solutions for different pathway lengths led to the conclusion that the optimal enzyme profiles followed a sequential pattern, in agreement with the experimental findings of Zaslaver et al. (2004).

To complement their experimental findings on pathway activation, Zaslaver et al. considered a model of a thermodynamically open pathway which incorporates a dynamic description of enzyme abundance with gene expression regulation. Considering an unbranched pathway with three reactions exhibiting Michaelis-Menten kinetics, the authors obtained (numerically) optimal enzymatic time profiles that qualitatively agree with the sequential behavior observed in their experiments.

In this paper we extend these studies on temporal distribution of enzymatic concentrations using a rigorous theoretical framework. In contrast to the numerical approaches previously developed in the literature (Klipp et al., 2002; Zaslaver et al., 2004), the optimization is tackled using analytical tools from optimal control theory (Pontryagin et al., 1962). We pose a dynamic optimization problem that accounts for time-resource optimality in the activation of thermodynamically open pathways under a constraint on the total enzyme abundance. The optimization inputs are the time-dependent enzyme concentrations required to drive the pathway from rest to a steady state characterized by a given metabolic flux. The analysis addresses unbranched pathways of arbitrary length and applies to a generic class of monomolecular enzyme kinetics that includes, but is not limited to, Mass Action, Michaelis-Menten, Hill, and some Power-law models.

The main result is the analytic derivation of an inherent sequential structure in the optimal activation. Each enzyme exhibits a profile which switches between zero and maximum concentration, following a temporal sequence that matches the order of the reaction steps in the pathway. These findings provide an analytic justification of the sequential behavior initially described in Klipp et al. (2002) and observed experimentally in Zaslaver et al. (2004), thus reinforcing the idea that sequential activation can be rationalized by means of an underlying optimal metabolic design. The use of an analytic approach allows the treatment of more general reaction kinetics than previous numerical investigations. Since the optimal activation sequence is invariant under a broad class of monomolecular kinetics, our result suggests that sequential enzyme expression may be a common feature of metabolic regulation, as it is a robust property of optimal pathway activation.

Other features of the optimized activation are also explored. Feasibility is addressed by deriving a general formula for the upper bound on the achievable target flux in terms of the saturation velocities of the individual reactions. Sensitivity analysis is performed by means of numerical solutions obtained from an equivalent nonlinear programming problem. These numerical results suggest that the optimized response is most sensitive to reactions located close to the beginning of the pathway, which is consistent with previous studies on sensitivity analysis (see, e.g., Klipp et al., 2005, p. 189). As a case study, we also investigate the effect of enzyme production dynamics by adding protein synthesis and degradation to the metabolic model. The extended model is optimized numerically 
and, although such kind of solution does not allow for generalizations, the result shows a temporal sequence that agrees with our theoretical analysis.

\section{Problem formulation}

\subsection{Model definition}

We consider unbranched metabolic pathways composed of irreversible reactions as in Fig. 1. In that scheme $x_{0}$ denotes the concentration of substrate feeding the pathway, $x_{i}(t)$ is the concentration of the $i$ th intermediate metabolite at time $t$ and $v_{i}$ is the rate of the $i$ th reaction. We assume that $v_{i}=v_{i}\left(x_{i}(t), u_{i}(t)\right) \geq 0$, where $u_{i}(t)$ is the concentration at time $t$ of the enzyme catalyzing the $i$ th reaction.

The pathway activity is presumed to have a negligible effect on the concentration of substrate, so $x_{0}$ is considered constant. The rate laws $v_{i}$ characterize the kinetic properties of the enzymes catalyzing the pathway. These are typically nonlinear in the metabolite concentrations $x_{i}$, so a general analysis is often not tractable. In this paper we consider a class of nonlinear monomolecular enzyme kinetics, namely those satisfying the following assumptions.

\section{Assumption 1.}

(A) The rate laws are linear in the enzyme concentrations, i.e., they can be written as

$$
v_{i}\left(x_{i}(t), u_{i}(t)\right)=w_{i}\left(x_{i}(t)\right) \cdot u_{i}(t), \quad i=0,1, \ldots, n,
$$

where $w_{i}\left(x_{i}(t)\right) \geq 0$ is continuous.

(B) The functions $w_{i}\left(x_{i}(t)\right)$ in (1) satisfy

$$
\begin{aligned}
& w_{i}(0)=0, \quad i=0,1, \ldots, n, \\
& \frac{d w_{i}}{d x_{i}}>0, \quad \text { for } x_{i}>0, i=0,1, \ldots, n .
\end{aligned}
$$

Assumption 1(A) is satisfied by most enzyme kinetic models (Cornish-Bowden, 2004a; Meléndez-Hevia et al., 1990), while (2) in Assumption 1(B) is trivial since a nonzero concentration $x_{i}(t)$ is required for the $i$ th reaction to occur. Equation (3) states that an increase in substrate $x_{i}$ yields an increase in the reaction rate, which can saturate for large substrate concentrations. This monotonicity condition is satisfied by a broad class of enzyme dynamics that includes, in particular, the following common kinetic models:

$$
\begin{aligned}
& w_{i}\left(x_{i}\right)=k_{i} x_{i}, \quad \text { (Mass Action) } \\
& w_{i}\left(x_{i}\right)=\frac{k_{i} x_{i}}{K_{i}+x_{i}}, \quad \text { (Michaelis-Menten) } \\
& w_{i}\left(x_{i}\right)=\frac{k_{i} x_{i}^{n}}{K_{i}+x_{i}^{n}}, \quad \text { (Hill) } \\
& w_{i}\left(x_{i}\right)=k_{i} x_{i}^{c}, \quad \text { (Power-law) }
\end{aligned}
$$

where $k_{i}>0, K_{i}>0, n \geq 0$ and $c>0$. 


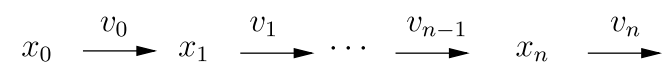

Fig. 1 Unbranched metabolic pathway.

The dynamic model for the pathway shown in Fig. 1 is given by mass conservation as

$$
\dot{x}_{i}(t)=v_{i-1}\left(x_{i-1}(t), u_{i-1}(t)\right)-v_{i}\left(x_{i}(t), u_{i}(t)\right), \quad i=1,2, \ldots, n .
$$

In this formulation the state vector of the model in (4) is composed of the metabolite concentrations, while the enzyme concentrations appear as time-dependent inputs. For future reference we define the state and input vectors as

$$
\begin{aligned}
& \mathbf{x}(t)=\left[\begin{array}{llll}
x_{1}(t) & x_{2}(t) & \cdots & x_{n}(t)
\end{array}\right]^{T}, \\
& \mathbf{u}(t)=\left[\begin{array}{llll}
u_{0}(t) & u_{1}(t) & \cdots & u_{n}(t)
\end{array}\right]^{T},
\end{aligned}
$$

respectively.

\subsection{Dynamic optimization problem}

We are interested in optimizing the transient dynamics of pathway activation. For clarity, we first give a precise definition of pathway activation and then we describe each element of the optimization problem itself: the cost function, the input constraints, and the terminal condition.

\subsubsection{Metabolic pathway activation}

Assuming that the pathway is initially inactive, i.e., $\mathbf{u}(0)=0, \mathbf{x}(0)=0$, we aim at obtaining temporal enzymatic profiles that drive the pathway to a steady state characterized by a prescribed constant flux $V>0$. From Fig. 1 and Eq. (4), the pathway reaches a steady state when

$$
v_{i}(t)=V, \quad \text { for } t \geq t_{f}, i=0,1, \ldots, n,
$$

where $t_{f}$ is the duration of the activation process whose value is left unspecified and regarded as an outcome of the optimization.

\subsubsection{Cost function}

If the pathway to be activated has a critical impact on cellular fitness, then the activation must build the metabolic product rapidly and with efficient enzyme usage. To quantitatively express this principle, $\mathbf{u}(t)$ should minimize a cost function of the form

$$
\mathcal{J}=\int_{0}^{t_{f}}\left(1+\boldsymbol{\alpha}^{T} \mathbf{u}(t)\right) d t,
$$

where the vector of weights $\boldsymbol{\alpha}$ is entry-wise nonnegative. The minimization of $\mathcal{J}$ implies a combined optimization of: (i) the time taken to reach the new steady state, and (ii) a measure of the enzyme usage. The weight vector $\boldsymbol{\alpha}$ can be appropriately tuned to reflect the relative biosynthetic cost of specific enzymes. If we choose $\alpha=0$ then $\mathcal{J}=t_{f}$, which 
corresponds to the total activation time. This measure of cost as the "true" time taken by the pathway activation contrasts with the approach in Klipp et al. (2002) whereby the activation duration for a thermodynamically closed pathway was measured by an averaged quantity known as the transition time (Llorens et al., 1999; Torres, 1994). The problem of minimizing the transition time within the framework of dynamic optimization has been addressed in Oyarzún et al. (2007).

\subsubsection{Input constraints}

The cell can expend only a limited set of resources on the activation of any given pathway. A simple and convenient way of taking those limitations into account is to consider an upper bound on the total enzyme abundance (Brown, 1991; Klipp et al., 2002). For that purpose, we impose the constraint that the components of the piece-wise continuous input functions $\mathbf{u}(t)$ lie in the simplex $\mathcal{U}$ defined by

$$
\mathcal{U}:\left\{\begin{array}{l}
\sum_{i=0}^{n} u_{i} \leq E_{T}, \\
u_{i} \geq 0, \quad i=0,1, \ldots, n,
\end{array}\right.
$$

where $E_{T}$ denotes the upper bound on enzymatic concentration. Potential important differences in the enzyme masses could be addressed by including weighting factors in the sum (7). To improve readability, such factors have not been incorporated in the subsequent analysis. Their inclusion has no impact on the main results, and leads to a straightforward scaling of the optimal solution.

\subsubsection{Terminal condition}

In principle, the terminal condition for the optimization problem is specified solely by enforcing the steady state after time $t_{f}$, which is described by (5). Once the pathway has reached the final metabolite levels (i.e., after time $t_{f}$ ), the steady state flux must be maintained by appropriate enzyme concentrations. From (1) and (5), it follows that the required steady state enzyme levels are

$$
u_{i}(t)=\frac{V}{w_{i}\left(x_{i}^{f}\right)}, \quad \text { for } t \geq t_{f}, i=0,1, \ldots, n,
$$

where $x_{i}^{f}=x_{i}\left(t_{f}\right)$ is the $i$ th component of the final state. Equation (8) specifies the steady state enzymatic concentrations that are needed to sustain the target flux. However, this condition alone does not ensure that the enzymatic levels are within the constraint region $\mathcal{U}$ after the optimization period. We need to ensure that the concentrations in (8) are feasible and thus, using (7) it follows that

$$
\mathcal{S}: \quad \sum_{i=0}^{n} \frac{V}{w_{i}\left(x_{i}^{f}\right)} \leq E_{T}, \quad x_{i}^{f}>0, i=0,1, \ldots, n .
$$

Terminal condition (9) guarantees that the steady state is compatible with the upper bound on total enzyme abundance. Rather than specifying the steady state as a single point, (9) defines a surface where the terminal steady state metabolite concentrations must lie.

In summary, the dynamic optimization problem reads as follows. 
Problem 1 (Optimal pathway activation). Consider the system (4) with $v_{i}$ satisfying Assumption $1, \mathbf{x}(0)=0$ and $V>0$. The problem is to find a terminal time $t_{f}>0$ and a piecewise continuous function $\mathbf{u}:\left[0, t_{f}\right] \rightarrow \mathcal{U}$, that minimizes the cost

$$
\mathcal{J}=\int_{0}^{t_{f}}\left(1+\boldsymbol{\alpha}^{T} \mathbf{u}(t)\right) d t,
$$

and drives the system to a steady state $\mathbf{x}\left(t_{f}\right) \in \mathcal{S}$.

\section{Optimal pathway activation}

In this section we present the sequential form of the solution to Problem 1 and illustrate it with an example.

\subsection{Form of the optimal activation}

Problem 1 is a nonlinear optimal control problem with free final time. A suitable framework for solving this kind of dynamic optimization problem is provided by Pontryagin's Minimum Principle (PMP) (Pontryagin et al., 1962). Application of PMP typically results in the statement of a two-point boundary value problem (BVP), so that any solution of the original optimization problem also solves the BVP. In general, solving this BVP can be very challenging and the analysis is typically carried out on a case-by-case basis.

Our main result describes qualitative features of the solution which can be obtained without solving the associated BVP. An explicit solution to Problem 1 is not attainable through PMP since the BVP does not admit a general solution. Even in a particular instance of the problem in which the pathway length and kinetics were specified, the resulting nonlinear dynamics would typically lead to a BVP which could only be treated numerically.

The main result of this paper is presented next. The proof can be found in Appendix A.

Theorem 1 (Form of the optimal activation). The optimal enzyme concentration profile $\mathbf{u}^{*}(t)$ for Problem 1 satisfies the following:

- At each time $t \in\left[0, t_{f}\right)$, only one enzyme is active (i.e., has nonzero concentration);

- The active enzyme is present at maximum concentration;

- Each enzyme is active over a single time interval;

- The order of enzyme activation matches the order of reactions in the pathway.

Formally, these conditions can be described as follows: There exists a set of switching times $\left\{t_{0}, t_{1}, \ldots, t_{n-1}\right\}$, with $0<t_{i}<t_{j}$ for $i<j$ and $t_{n-1}=t_{f}$ which partition the optimization interval as $\left[0, t_{f}\right)=\left[0, t_{0}\right) \cup\left[t_{0}, t_{1}\right) \cup \cdots \cup\left[t_{n-2}, t_{n-1}\right)$, such that the optimal profile of the ith enzyme satisfies

$$
u_{i}^{*}(t)= \begin{cases}E_{T}, & \text { for } t \in T_{i}, \\ 0, & \text { for } t \notin T_{i},\end{cases}
$$

where $T_{0}=\left[0, t_{0}\right)$ and $T_{i}=\left[t_{i-1}, t_{i}\right)$ for $i=1,2, \ldots, n-1$. 
Equation (10) shows that the optimal enzyme profiles switch between 0 and the maximal level $E_{T}$. Such profiles are called "bang-bang" inputs in control engineering, and are a common feature of solutions in classical time optimal control (Pontryagin et al., 1962). The bang-bang quality of the solutions to Problem 1 is a consequence of the geometry of the constraint region $\mathcal{U}$ and the fact that the dynamics and the cost depend linearly on the input $\mathbf{u}(t)$. We emphasize that the optimization is carried out over all piece-wise continuous concentration profiles. The piece-wise constant form of the optimal input is a consequence of the optimization, not an a priori constraint on the inputs. It is also observed that the form of the optimal solution does not depend on the weight $\boldsymbol{\alpha}$. Therefore, variations in the biosynthetic costs for enzyme production will be reflected only in the activation duration of the individual reactions, without any effect in the activation sequence. The value of $\boldsymbol{\alpha}$ will also affect the final time $t_{f}$ which is not prespecified, but is an outcome of the optimization procedure.

The sequential nature of the activation profile has been shown numerically in Klipp et al. (2002) for thermodynamically closed pathways with linear kinetics and specific lengths. Theorem 1 extends this finding of sequential behavior to a much broader class of reaction kinetics. Moreover, the proof reveals how the sequential property emerges from the optimality principle. We find that the activation sequence is a consequence of both the pathway structure and the reaction kinetics. From an intuitive point of view, the "pipeline" structure of the pathway implies the $i$ th metabolite cannot be produced unless the upstream portion of the pathway has been activated. Moreover, the monotonicity condition on the kinetics (3) precludes the optimality of activating an upstream reaction after the $i$ th one has already been activated (a fact that arises from (A.24) and (A.25)). The generality of Theorem 1 indicates that this sequential behavior is a robust feature of time-resource optimal pathway activation.

Before presenting a concrete example, we observe that the qualitative description of the optimal solution provided by Theorem 1 considerably simplifies the computation of the optimal solutions, since one needs only to optimize over the $n$ switching times $\left\{t_{0}, t_{1}, \ldots, t_{n-1}\right\}$, rather than over the whole class of admissible inputs.

\subsection{Example}

As an illustrative example, we consider a pathway as in Fig. 1 of length $n=3$, where all the reactions exhibit Michaelis-Menten kinetics of the form

$$
v_{i}\left(x_{i}\right)=\frac{k_{\mathrm{cat} i} x_{i}(t)}{K_{m i}+x_{i}(t)} u_{i}(t) .
$$

The model parameters are $k_{\text {cat } 1}=1 \mathrm{~s}^{-1}, k_{\text {cat } 2}=2 \mathrm{~s}^{-1}, k_{\text {cat } 3}=4 \mathrm{~s}^{-1}, k_{\text {cat } 4}=3 \mathrm{~s}^{-1}$, $K_{m i}=1 \mathrm{mM}, x_{0}=5 \mathrm{mM}$ and we set the enzymatic weights to $\alpha_{i}=1 \mathrm{mM}^{-1} \mathrm{~s}$. The optimal enzyme and metabolite profiles for Problem 1 with $V=0.2 \mathrm{mM} \mathrm{s}^{-1}$ are shown in Fig. 2. The optimal control problem was recast as a nonlinear optimization program and solved with the gradient-based routine fmincon available in the Optimization Toolbox for Matlab. ${ }^{1}$ Enzyme levels are in units of $E_{T}=1 \mathrm{mM}$ and metabolites are in units of $K_{m}=1 \mathrm{mM}$. The optimal switchings occur at $t_{0}=1.5 \mathrm{~s}, t_{1}=2.1 \mathrm{~s}$ and $t_{2}=2.4 \mathrm{~s}$, and

\footnotetext{
${ }^{1}$ Matlab $^{\circledR}$ is a registered trademark of The Mathworks.
} 


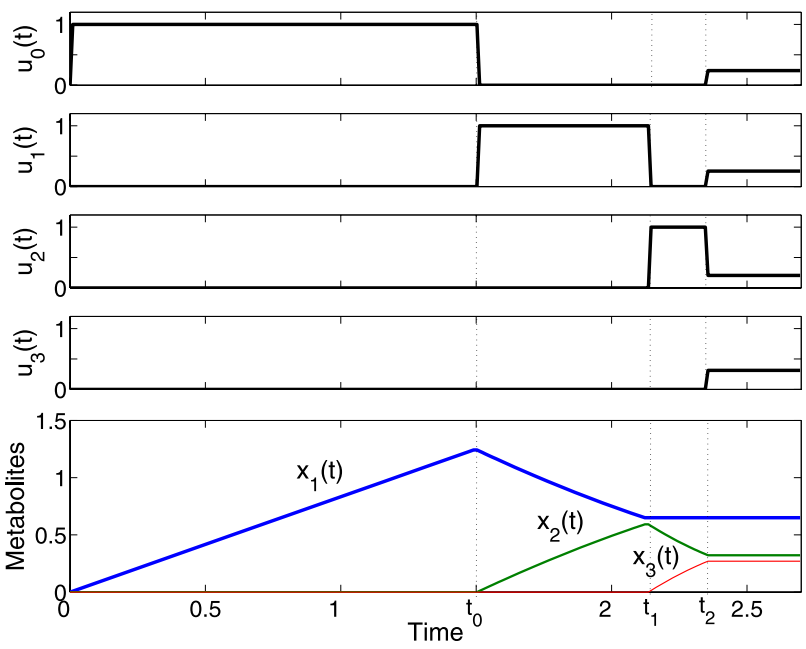

Fig. 2 Optimal activation for pathway of length $n=3$ with Michaelis-Menten kinetics.

the steady state concentrations of the metabolites are $x_{1}^{f}=0.65 \mathrm{mM}, x_{2}^{f}=0.32 \mathrm{mM}$ and $x_{3}^{f}=0.29 \mathrm{mM}$. The enzyme profiles satisfy all the properties in Theorem 1 and guarantee that the steady state is maintained after the activation time $t \geq t_{f}=t_{2}$. The terminal steady state enzyme levels are computed directly from (8). We also notice that the last enzyme needs to be present only after the activation period, which is required to achieve the steady state flux.

\section{Further analysis}

\subsection{Maximal steady state flux}

The flux achievable by the pathway is constrained by the bound on the total enzyme abundance (7) and the saturating velocities of the reaction steps. From (1), we define the saturating velocities, denoted $\hat{w}_{i}$, as

$$
\hat{w}_{i}=\sup _{x_{i}>0} w_{i}\left(x_{i}\right)=\sup _{x_{i}>0} v_{i}\left(x_{i}, 1\right) .
$$

From (9) it follows that the achievable flux is upper-bounded by

$$
\hat{V}=E_{T}\left(\sum_{i=0}^{n} \frac{1}{\hat{w}_{i}}\right)^{-1},
$$

where we interpret $\frac{1}{\infty}=0$ for the case of non-saturating kinetics (e.g., Mass Action steps - such reactions do not constrain the achievable flux). Equation (13) gives the maximal flux under which the optimization problem is feasible. This formula also indicates how the total enzyme pool should be distributed to achieve maximal flux. Flux $\hat{V}$ will 
only be reached if $\frac{E_{T}}{\hat{w}_{i}}\left(\sum_{i=0}^{n} \frac{1}{\hat{w}_{i}}\right)^{-1}$ enzymatic activity is dedicated to enzyme $u_{i}$. In the typical case that the saturating velocities in (12) are not attained at finite metabolite concentrations, the upper bound $\hat{V}$ is not an achievable target. In such cases it can be shown that as the target flux $V$ approaches the value $\hat{V}$ the optimal cost grows unbounded. The supremal flux $\hat{V}$ could only be reached by saturating all the reactions in the pathway, which in turn would require an infinite activation period.

\subsection{Solution sensitivity}

The sensitivity properties of the optimal solution are presented through case studies. We consider pathways of length $n=6$ with $x_{0}=1 \mathrm{mM}$ and assume that all the reactions follow Michaelis-Menten kinetics of the form (11). We adopt as nominal model parameters $k_{\text {cat } i}=1 \mathrm{~s}^{-1}$ and $K_{m i}=1 \mathrm{mM}$. The nominal values for the enzyme weights are chosen as $\alpha_{i}=5 \mathrm{mM}^{-1} \mathrm{~s}$ and the numerical solutions are obtained as in Section 3.2.

\subsubsection{Sensitivity to kinetic parameters}

In order to study the effect of kinetic parameters on the optimal activation, we compare the sensitivity of the optimal cost with respect to parameters $k_{\text {cat } i}$ and $K_{m i}$ of each reaction. Varying one constant at a time and setting the others to their nominal values, we obtain optimal solutions for different values of $k_{\text {cat } i}$ and $K_{m i}$ in a range of $\pm 90 \%$ of their nominal values. The target flux is chosen as $80 \%$ of the maximal flux $\hat{V}$ (see Eq. (13)) for the parameter range. The results are shown in Fig. 3, where the optimal cost normalized with respect to its nominal value is shown for $k_{\text {cat } i}$ between $10 \%$ and $25 \%$ of the nominal value, and $K_{m i}$ from $10 \%$ to $100 \%$ of its nominal value.

As expected the optimal activation takes longer as parameters $k_{\text {cat } i}$ decrease. As shown in Fig. 3, the optimal activation is less sensitive to the $k_{\text {cat }}$ parameter of those reactions that are located toward the end of the pathway. For example, reducing $k_{\text {cat } 6}$ to $10 \%$ of its nominal value yields a five-fold increase in the optimal cost, whereas the same reduction in $k_{\text {cat } 1}$ yields almost an eight-fold increase. This is a consequence of the fact that early reactions must process more material in order to reach steady state. The overall trend is consistent with the commonly accepted assertion (Klipp et al., 2005) in the literature on
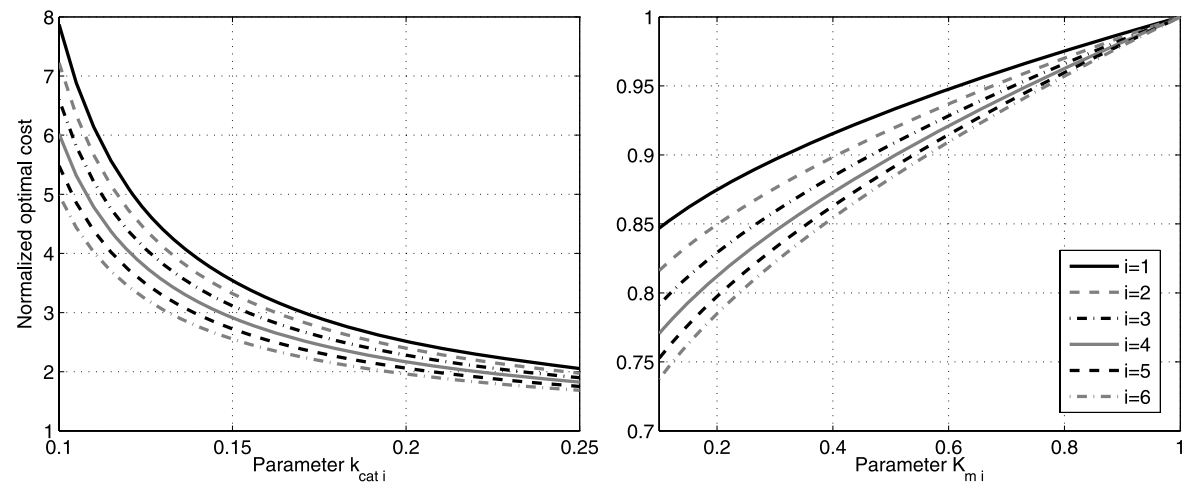

Fig. 3 Normalized optimal cost as a function of the kinetic parameters (in units of their nominal values). 
Metabolic Control Analysis that the sensitivity of the steady state flux with respect to a particular $k_{\mathrm{cat} i}$ decreases as the reaction is located toward the end of the pathway.

The sensitivity with respect to parameters $K_{m i}$ follows the opposite trend, with increased sensitivity later in the pathway. This conclusion has more to do with individual kinetics than with the behavior of the system as a whole. As mentioned, the first reactions process more material and so operate at higher substrate concentrations than those downstream. The saturating nature of the Michaelis-Menten kinetic means that those reactions operating at high substrate concentrations are less susceptible to variation in $K_{m}$.

\subsubsection{Sensitivity to enzyme weighting}

As mentioned in Section 2.2, the weighting vector $\boldsymbol{\alpha}$ allows the optimization procedure to reflect the relative biosynthetic costs of the enzymes in the pathway. To explore the sensitivity of the optimal activation with respect to the enzyme weighting, we consider the effect of $\alpha_{i}$ on the pulse width of enzyme $u_{i}$ : the length of the time interval during which enzyme $u_{i}$ is active. Changing one enzyme weight at a time, we compute optimal solutions for $\alpha_{i}$ in the range $\pm 50 \%$ of the nominal value with a target flux of $80 \%$ of the maximum $\hat{V}$. The optimal pulse width normalized with respect to its nominal value is shown in Fig. 4.

It can be observed that as an enzyme is more strongly penalized, its optimal pulse width is reduced. The reduction is larger for those enzymes acting close to the end of the pathway. This implies that significant reductions in the use of early enzymes can be only be achieved with very large weights, while more freedom is available for the ones toward the end of the pathway. For example, for enzyme $u_{1}$ only a marginal reduction can be achieved with a $50 \%$ increase in the weight, while for $u_{5}$ a reduction over $10 \%$ can be attained. This is a consequence of the pathway structure and suggests, as in the previous case study, that the importance of a specific enzyme in the activation dynamics is a decreasing function of its position in the pathway.

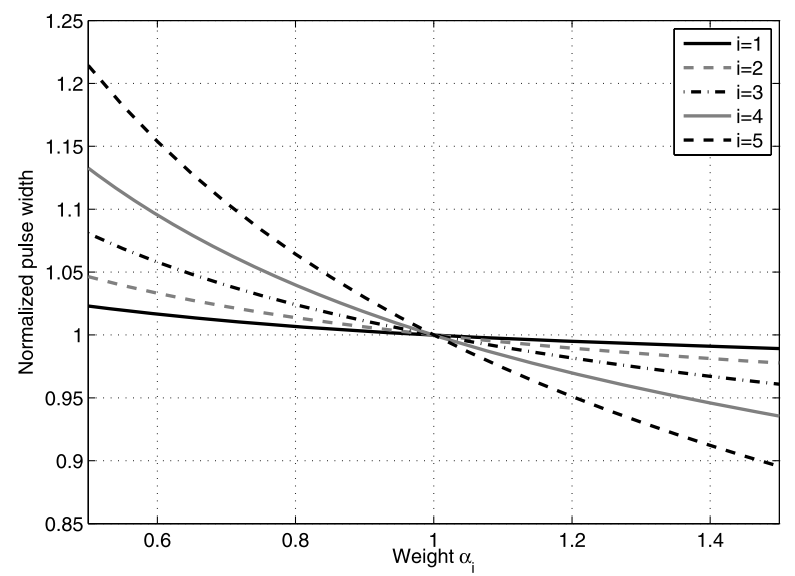

Fig. 4 Normalized optimal pulse width of each enzyme as a function of the enzyme weight $\alpha_{i}$ (in units of their nominal values). 


\subsection{Effect of enzyme production dynamics}

Enzyme synthesis cannot be as fast as required by the switching profiles that solve Problem 1 . More realistic solutions can be obtained by extending the model to include enzyme production dynamics. The production of enzyme $u_{i}$ can be described by

$$
\dot{u}_{i}(t)=r_{i}(t)-\lambda u_{i}(t)
$$

where $r_{i}(t)$ is the expression rate of $u_{i}(t)$ and $\lambda$ accounts for dilution by cell growth and the constituent protein degradation rate. As an illustration, we review the example of Section 3.2 with this extended formulation. Instead of optimizing with respect to the enzymatic levels, we consider the metabolic model extended with the enzyme production model shown in (14), and optimize over the enzyme expression rates. The expression rates are bounded as $0 \leq r_{i}(t) \leq 1 \mathrm{mM} \mathrm{s}^{-1}$ and we set $\lambda=0.5 \mathrm{~s}^{-1}$. The constraint in total enzyme abundance is replaced by box constraints of the form $0 \leq u_{i}(t) \leq E_{T}$ and we fix the steady state to match the one shown in Fig. 2. The model parameters and the cost function are chosen identical to the ones in Section 3.2.

The dynamic optimization problem was numerically solved with the pseudospectral optimal control solver Tomlab/PROPT (Rutquist and Edvall, 2009). The optimal expression rates are shown in Fig. 5, whereas the corresponding enzyme and metabolite profiles are shown in Fig. 6. To facilitate the comparison with the results of Section 3.2, the enzyme profiles of Fig. 2 are included in dashed line in Fig. 6. The optimal expression rates follow a switching pattern that matches the pathway topology, which lead to enzyme profiles that follow a sequential activation similar to the one discussed in this paper. The $\mathrm{ON} / \mathrm{OFF}$ behavior seen in the expression rates is consistent with boolean models for genetic networks. Such models have been widely used for the analysis of gene expression networks (Chaves et al., 2005). On the other hand, from Fig. 6 we see that by including the enzyme production model, the switching profiles of Fig. 2 become continuous functions of time and hence lead to more realistic optimal responses.

\section{Discussion}

Metabolic activity is regulated to accommodate resource allocation and product formation in the face of varying external conditions. This regulation is implemented through the

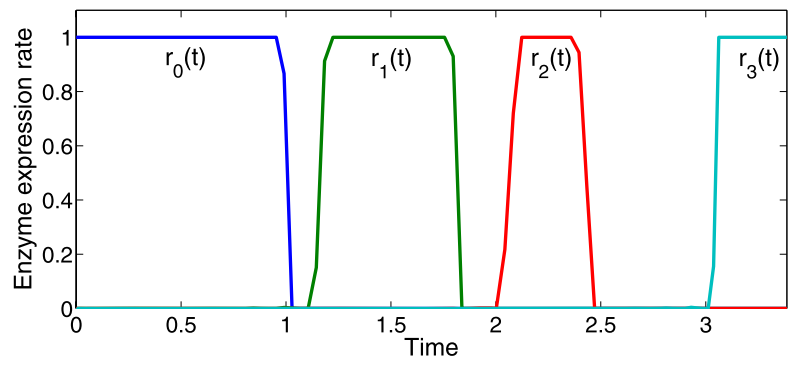

Fig. 5 Optimal expression rates for pathway of Section 3.2 extended with enzyme production dynamics. 

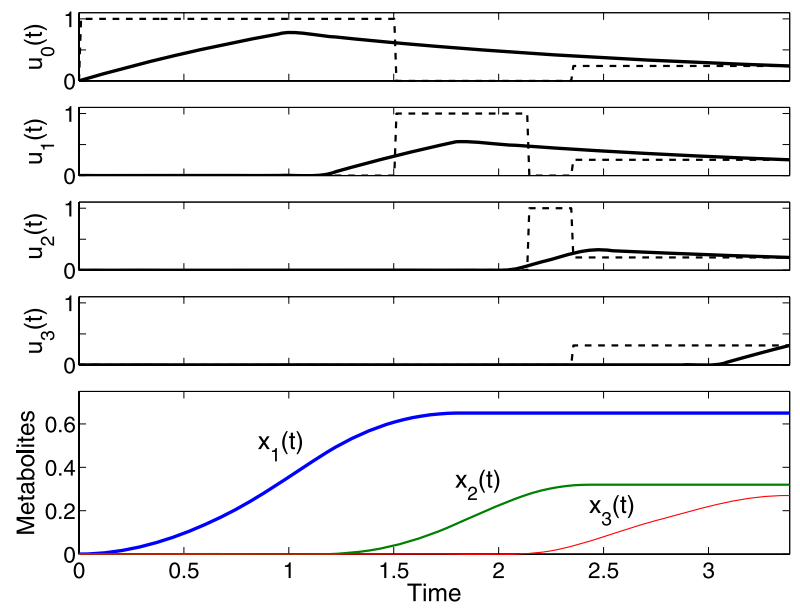

Fig. 6 Optimal enzyme and metabolite concentrations for pathway of Section 3.2 extended with enzyme production dynamics. Enzyme profiles of Fig. 2 are shown in dashed line.

genetic control of enzyme expression. In this paper we study such a control policy for the case of pathway activation under the premise that it satisfies a time-resource optimality criterion. Under constraints on the total enzyme availability, we optimize a set of timedependent enzyme concentrations that drive the pathway to a target steady state flux.

As a consequence of both reaction kinetics and pathway topology, a well defined temporal program in the optimal enzyme levels is revealed. The profile of each enzyme switches between zero and maximum concentration following a temporal ordering that matches the pathway topology. The problem is stated in a control theoretic framework and the analysis is performed with tools from Optimal Control Theory (Pontryagin et al., 1962). This allows treatment of more general reaction kinetics than previously considered in the literature (Klipp et al., 2002; Zaslaver et al., 2004). Our analytical results assume no specific kinetics or pathway length, and hold independently of the parameter values. Since standard kinetics (Mass Action, Michaelis-Menten, Hill, and some Power-law) satisfy the required assumptions, the results presented here extend those in Klipp et al. (2002).

The switching nature of the optimal enzymatic profiles allows us to recast the dynamic optimization as a static nonlinear programming problem which can be solved with available numerical algorithms. The decision variables in this nonlinear program are the switching times of each optimal profile, and the optimization is carried out under the positivity constraints in both enzyme and metabolite concentrations. The terminal condition (9) defines the set of feasible steady states compatible with the constraint on the total amount of enzyme. In other optimization approaches, such as Flux Balance Analysis (Varma and Palsson, 1994) and multiobjective optimization (Vera et al., 2003), the constraints on steady state concentrations and metabolic fluxes are specified individually. A distinctive feature of a constraint such as (9) is that, in accounting for the limitation in total enzyme abundance, the steady state of the metabolites and flux are considered simultaneously.

Despite the ability of this framework to account for more general kinetics than previous efforts (Klipp et al., 2002; Zaslaver et al., 2004), at present we have only been able 
to complete this analysis with a very simplified description of enzyme dynamics. An improved framework was considered for Michalis-Menten kinetics in Zaslaver et al. (2004) by including transcriptional feedback in the model. Enzyme levels were set to be dependent on the metabolic product and thus the optimization was carried out over the feedback strengths. In our case the enzyme profiles are considered as independent functions of time and optimized over the class of piece-wise continuous functions. This allows discontinuous profiles to be identified as optimal. The result is an activation scheme in which the enzyme concentrations vary more quickly than the metabolite concentrations, when in fact the reverse is a more accurate description of cellular events. This could be addressed by including the rate of change of the enzyme concentrations $\dot{u}(t)$ in the cost function. This is a standard approach in control engineering and has recently been used in the context of homeostatic regulation as well (Uygun et al., 2006).

Another way to account for this is by extending the model with enzyme production dynamics. Pathway optimization can then be carried out by finding expression rates that minimize a meaningful metabolic objective. The optimization is not only subject to constraints in enzyme and metabolite levels, but bounds on the expression rates should also be included. As suggested by the example of Section 4.3, the optimal activation of the extended model can follow the same temporal pattern as the one obtained from our theoretical analysis. However, the numerical nature of the solution prevents us from characterizing this behavior as a general principle. This extended formulation allows the derivation of numerical solutions, but presents major challenges for a general analysis and is the subject of ongoing research. Similar considerations arise when considering pathways with more complicated metabolic interactions such as product inhibition or allostery.

In our efforts to develop a theoretical foundation for the sequential activation of metabolic pathways, the analysis has been limited to unbranched pathways. Sequential activation was experimentally shown in Zaslaver et al. (2004) for the Arginine pathway in $E$. coli. It was detected in each branch of the pathway, but no clear relation between the activation of adjacent branches was identified. Extensions of our methodology to branched pathways are not straightforward; in our formulation all the available protein is allocated to a single reaction at a time, which is not realistic when different branches are working simultaneously. It seems that the study of branched pathways should consider different enzymatic constraints and, possibly, a different cost function. Nevertheless, complex topologies are a challenging scenario for other cellular processes in which optimization may play an important role, such as cellular growth (Mahadevan et al., 2002) and homeostatic regulation (Uygun et al., 2006).

\section{Conclusions}

In this paper we have presented a theoretical analysis of a dynamic optimization problem arising from the activation of metabolic pathways. Unlike numerical approaches, the theoretical nature of this study allows us to identify the optimal responses as features underpinning the dynamics of the metabolic model. This line of study holds promise for the identification of design principles in metabolic regulation, mainly because a theoretical approach reveals them as structural properties of the network, rather than attributes achieved through fine tuning of the model parameters. A major obstacle is that the optimization becomes analytically intractable for many cases of practical importance, and 
therefore one must usually resort to numerical solutions. Nonetheless, despite a number of simplifications in the considered model, the ideas presented here reveal principles behind the dynamics of metabolic regulation.

Static optimization methods such as Flux Balance Analysis have had great success in aiding both scientific investigations and engineering applications in metabolism. These techniques could be complemented with a sound theory of dynamic optimization to provide deeper insights into metabolic dynamics. Such a combination can be conceived as a two-stage optimization process: Once optimal steady state fluxes are identified, the transient responses are optimized to meet additional metabolic objectives under the constraint of reaching the optimal flux distribution. Since the optimality criteria used in both stages are of different nature, this synergy can yield a more comprehensive approach to the investigation of metabolic pathways. However, it must be pointed out that given the large scale of real metabolic networks, the use of dynamic optimization as a practical tool still requires the development of appropriate computational techniques that can efficiently cope with high-dimensional problems, perhaps in the spirit of some of the recent work in the field (Mahadevan et al., 2002; Uygun et al., 2006; Banga et al., 2005).

\section{Acknowledgements}

This work was supported by Science Foundation Ireland (SFI) under Research Professor Award 03/RP1/I382.

\section{Appendix A: Proof of Theorem 1}

Pontryagin's Minimum Principle (Pontryagin et al., 1962) provides a set of necessary conditions that must be satisfied by the solution of the optimal control problem. For that purpose, the scalar Hamiltonian is defined as

$$
\mathcal{H}(\mathbf{x}(t), \mathbf{u}(t), \mathbf{p}(t))=1+\boldsymbol{\alpha}^{T} \mathbf{u}(t)+\mathbf{p}(t)^{T} \dot{\mathbf{x}}(t),
$$

where the vector $\mathbf{p}(t)=\left[p_{1}(t), p_{2}(t), \ldots, p_{n}(t)\right]^{T}$ is called the system's co-state and $\dot{\mathbf{x}}(t)$ denotes the dynamics (4) in vector form. From (1) and (4), the Hamiltonian function can be written as

$$
\mathcal{H}(\mathbf{x}(t), \mathbf{u}(t), \mathbf{p}(t))=1+\sum_{i=0}^{n} h_{i}(t) u_{i}(t),
$$

where the function $h_{i}(t)$ is called the $i$ th switching function and is given by

$$
h_{i}(t)=\alpha_{i}+\left(p_{i+1}(t)-p_{i}(t)\right) w_{i}\left(x_{i}(t)\right), \quad \forall i=0,1, \ldots, n,
$$

where we define, for convenience of notation, $p_{0}(t)=0$ and $p_{n+1}(t)=0$. The proof hinges in the following statements of PMP: 
- The optimal control input $\mathbf{u}^{*}(t)$ minimizes the Hamiltonian for all $t \in\left[0, t_{f}\right)$, i.e.,

$$
\mathcal{H}\left(\mathbf{x}^{*}(t), \mathbf{u}^{*}(t), \mathbf{p}^{*}(t)\right)=\min _{\mathbf{u}(t) \in \mathcal{U}} \mathcal{H}\left(\mathbf{x}^{*}(t), \mathbf{u}(t), \mathbf{p}^{*}(t)\right) .
$$

- The trajectory of the optimal co-state vector satisfies

$$
\dot{\mathbf{p}}^{*}(t)=-\frac{\partial \mathcal{H}\left(\mathbf{x}^{*}(t), \mathbf{u}^{*}(t), \mathbf{p}^{*}(t)\right)}{\partial x}, \quad \forall t \in\left[0, t_{f}\right) .
$$

From (4) and (A.1), using (A.5) the optimal trajectory of the $i$ th co-state is given by

$$
\dot{p}_{i}(t)=\left(p_{i}(t)-p_{i+1}(t)\right) \frac{\partial w_{i}}{\partial x_{i}} u_{i}(t), \quad \forall i=1,2, \ldots, n .
$$

- The Hamiltonian evaluated along the optimal trajectory is zero for all $t \in\left[0, t_{f}\right)$, that is,

$$
\mathcal{H}\left(\mathbf{x}^{*}(t), \mathbf{u}^{*}(t), \mathbf{p}^{*}(t)\right)=0, \quad \forall t \in\left[0, t_{f}\right] .
$$

Denote the set of vertices of $\mathcal{U}$ as $\mathcal{V}=\left\{\mathbf{e}_{0}, \mathbf{e}_{1}, \ldots, \mathbf{e}_{n}\right\} \cup\{\mathbf{0}\}$, where $\mathbf{e}_{i}$ has $E_{T}$ in its $(i+1)$ st entry and 0 elsewhere. Similarly, the set of $n$-dimensional faces of $\mathcal{U}$ is defined as $\mathcal{F}=\left\{F_{0}, F_{1}, \ldots, F_{n}\right\} \cup\{\mathcal{P}\}$, where $F_{i}$ and $\mathcal{P}$ are the faces defined by the hyperplanes $F_{i}=\left\{\mathbf{u}(t) \in \mathcal{U}: u_{i}(t)=0\right\}$ and $\mathcal{P}=\left\{\mathbf{u}(t) \in \mathcal{U}: \sum_{i=0}^{n} u_{i}(t)=E_{T}\right\}$, respectively. We notice from (A.2) that $\mathcal{H}(\mathbf{x}(t), \mathbf{u}(t), \mathbf{p}(t))$ is a linear function defined over the simplex $\mathcal{U}$. Therefore, from (A.4) it follows that the optimal control is located in the boundary of $\mathcal{U}$ for all $t \in\left[0, t_{f}\right)$ and, moreover, it holds that $\mathbf{u}^{*}(t) \in \mathcal{V}, \forall t \in\left[0, t_{f}\right)$. This means that the optimal control can always be found in the set of vertices of $\mathcal{U}$. However, if the optimal control is not unique then it will lie in a face of the simplex $\mathcal{U}$, that is, in the convex hull of a subset of $\mathcal{V}$. We next present a simple fact that will be needed later to show that the optimal control is indeed located only in one vertex at a time.

Fact 1. Let $f(\mathbf{u}): \mathcal{U} \rightarrow \mathbb{R}$ be a linear function of $\mathbf{u}$ with $\mathcal{U}$ defined in (7). Then, if vertex $\mathbf{e}_{j}$ is not minimal, then any point where $f(\mathbf{u})$ attains its minimum cannot be located in a face of $\mathcal{U}$ that contains $\mathbf{e}_{j}$.

Proof: The proof follows by contradiction. Let $Q$ be any $r$-dimensional face of $\mathcal{U}$ with vertex set $\mathcal{V}_{Q} \subseteq \mathcal{V}$. Suppose $\mathcal{V}_{Q}$ is partitioned in sets containing the minimal and nonminimal vertices, $\mathcal{V}_{Q+}$ and $\mathcal{V}_{Q-}$, respectively. Let $\mathbf{e}_{j} \in \mathcal{V}_{Q-}$ and assume that there exists $\mathbf{y} \in Q$ such that $\mathbf{y}$ is minimal. Then, if we define the index sets $\mathbb{I}_{Q+}=\{i \in\{0,1, \ldots, n\}$ : $\left.\mathbf{e}_{i} \in \mathcal{V}_{Q_{+}}\right\}$and $\mathbb{I}_{Q-}=\left\{i: \mathbf{e}_{i} \in \mathcal{V}_{Q_{-}}\right\}$, there exists $\lambda_{i} \geq 0$ such that

$$
\mathbf{y}=\sum_{i \in \mathbb{I}_{Q-}} \lambda_{i} \mathbf{e}_{i}+\sum_{i \in \mathbb{I}_{Q+}} \lambda_{i} \mathbf{e}_{i},
$$

where $\sum_{i \in \mathbb{I}_{Q-} \cup \mathbb{I}_{Q+}} \lambda_{i}=1$. Linearity of $f(\mathbf{u})$ implies that $f(\mathbf{y})=f\left(\mathbf{e}_{i}\right), \forall i \in \mathbb{I}_{Q+}$, and (A.8) yields

$$
\begin{aligned}
& \left(1-\sum_{i \in \mathbb{I}_{Q+}} \lambda_{i}\right) f(\mathbf{y})=\sum_{i \in \mathbb{I}_{Q-}} \lambda_{i} f\left(\mathbf{e}_{i}\right), \\
& \sum_{i \in \mathbb{I}_{Q_{-}}} \lambda_{i} f(\mathbf{y})=\sum_{i \in \mathbb{I}_{Q-}} \lambda_{i} f\left(\mathbf{e}_{i}\right),
\end{aligned}
$$


which is a contradiction since by hypothesis $f(y)<f\left(\mathbf{e}_{i}\right)$ for all $i \in \mathbb{I}_{Q_{-}}$.

From (A.7) we have that $\mathcal{H}$ must vanish along the optimal trajectory, which together with (A.2) implies that $\mathbf{u}^{*}(t) \neq \mathbf{0}, \forall t \in\left[0, t_{f}\right)$. This precludes the optimality of the origin, and thus we can use Fact 1 and (A.4) to conclude that $\mathbf{u}^{*}(t) \notin F_{i} \backslash \mathcal{V}, \forall i$, so that $\mathbf{u}^{*}(t) \in \mathcal{P}$ and therefore the optimal solution satisfies

$$
\sum_{i=0}^{n} u_{i}^{*}(t)=E_{T} .
$$

Suppose $\left\{T_{0}, T_{1}, \ldots, T_{q}\right\}$ is a partition of the interval $\left[0, t_{f}\right)$ such that $T_{0}=\left[0, t_{0}\right)$ and $T_{i}=\left[t_{i-1}, t_{i}\right), \forall i=1,2, \ldots, q$, with $t_{i}<t_{j}, \forall i<j$ and $t_{q}=t_{f}$. Let $U_{\ell}^{*}=\left\{u_{i}\right\}, i \in \mathbb{I}_{\ell}$, be the set of nonzero enzymes of the optimal solution in the interval $T_{\ell}$, that is, the optimal control $\mathbf{u}^{*}(t)$ lies in the convex hull of vertexes $\left\{\mathbf{e}_{j}\right\}, j \in \mathbb{I}_{\ell}, \forall t \in T_{\ell}$. This implies that the nonzero enzymes satisfy

$$
\sum_{i \in \mathbb{I}_{\ell}} u_{i}(t)=E_{T}, \quad \forall t \in T_{\ell}
$$

We consider a partition $\left\{T_{0}, T_{1}, \ldots, T_{q}\right\}$ such that $U_{i}^{*} \neq U_{i+1}^{*}, \forall i=0,1, \ldots, q-1$. In this setup, the proof follows by showing that

$$
\begin{aligned}
& U_{\ell}^{*}=\left\{u_{\ell}\right\}, \quad \forall \ell=0,1, \ldots, q, \\
& q=n-1 .
\end{aligned}
$$

The general idea behind the proof is that the system structure and dynamics force well defined behaviors in the time courses of the switching functions in (A.2). As a first step, we can see that by using (A.7) and (A.2) it follows that for all $t \in\left[0, t_{f}\right)$ there exists $i \in\{0,1, \ldots, n\}$ such that $h_{i}(t)<0$, which together with (A.4) implies that

$$
h_{j}(t)=\min \left\{h_{0}(t), h_{1}(t), \ldots, h_{n}(t)\right\}, \quad \forall j \in \mathbb{I}_{\ell}, \forall t \in T_{\ell} .
$$

Combining (A.2), (A.7), (A.11), and (A.14) implies that the switching function corresponding to each nonzero enzyme is given by

$$
h_{j}(t)=-\frac{1}{E_{T}}<0, \quad \forall j \in \mathbb{I}_{\ell}, \forall t \in T_{\ell}
$$

We note from (2) and (9) that $\mathbf{x}\left(t_{f}\right) \neq 0$, which using the fact that $\mathbf{x}(0)=0$ implies that each $u_{i}(t)$ must be active for some nonempty interval, i.e., for each $u_{i}, i=0,1, \ldots, n$, there exists an interval $R_{i} \neq \emptyset$ such that

$$
u_{i}(t) \neq 0, \quad \forall t \in R_{i}
$$

The proof follows using an inductive procedure based on the following result. 
Fact 2. Consider interval $T_{\ell}, \ell \geq 2$, and assume that

$$
\begin{array}{ll}
x_{i}\left(t_{\ell}\right)=0, & \forall i>\ell+1, \\
U_{j}^{*}=\left\{u_{j}\right\}, & \forall j \leq \ell .
\end{array}
$$

Then,

$$
U_{\ell+1}^{*}=\left\{u_{\ell+1}\right\} .
$$

Proof:

We first note that since $\mathbf{x}(0)=0$ and $w_{i}(0)=0$, then (A.18) implies

$$
x_{j}(t)=0, \quad \forall t \in \bigcup_{i=0}^{j-2} T_{i}, \forall 2 \leq j \leq \ell,
$$

which combined with (A.3) yields

$$
h_{j}(t)=\alpha_{j}, \quad \forall t \in \bigcup_{i=0}^{j-2} T_{i}, \quad \forall 2 \leq j \leq \ell .
$$

From (4), (A.3), and (A.6), for all $j$ it holds

$$
\begin{aligned}
\dot{h}_{j}(t)= & \left(\dot{p}_{j+1}(t)-\dot{p}_{j}(t)\right) w_{j}\left(x_{j}(t)\right)+\left(p_{j+1}(t)-p_{j}(t)\right) \frac{\partial w_{j}}{\partial x_{j}} \dot{x}_{j}(t) \\
= & \left(p_{j+1}(t)-p_{j+2}(t)\right) \frac{\partial w_{j+1}}{\partial x_{j+1}} w_{j}\left(x_{j}(t)\right) u_{j+1}(t) \\
& -\left(p_{j}(t)-p_{j+1}(t)\right) \frac{\partial w_{j}}{\partial x_{j}} w_{j-1}\left(x_{j-1}(t)\right) u_{j-1}(t),
\end{aligned}
$$

where we define $w_{-1}=0$. Since $u_{j}(t)=0, \forall j \neq \ell, \forall t \in T_{\ell}$, (A.21) yields

$$
\dot{h}_{j}(t)=0, \quad \forall j \notin\{\ell-1, \ell+1\}, \quad \forall t \in T_{\ell} .
$$

On the other hand, if $j=\ell-1$ then $u_{j+1}(t)=E_{T}, \forall t \in T_{\ell}$ and $u_{j-1}(t)=0$, which after substituting in (A.21) yields

$$
\dot{h}_{\ell-1}(t)=\left(p_{\ell}(t)-p_{\ell+1}(t)\right) \frac{\partial w_{\ell}}{\partial x_{\ell}} w_{\ell-1}\left(x_{\ell-1}(t)\right) E_{T}, \quad \forall t \in T_{\ell} .
$$

Combining (A.23) and (A.3) with $i=\ell$ leads to

$$
\dot{h}_{\ell-1}(t)=\left(\frac{\alpha_{\ell}-h_{\ell}(t)}{w_{\ell}\left(x_{\ell}(t)\right)}\right) \frac{\partial w_{\ell}}{\partial x_{\ell}} w_{\ell-1}\left(x_{\ell-1}(t)\right) E_{T}, \quad \forall t \in T_{\ell} .
$$

Equation (A.3) with $i=\ell$ implies that $w_{\ell}(t)>0, \forall t \in T_{\ell}$, since otherwise $h_{\ell}(t)=\alpha_{\ell} \geq 0$ for some $t \in T_{\ell}$ and (A.15) cannot be satisfied. This guarantees that $\dot{h}_{\ell-1}(t)$ in (A.24) is 
well defined in the interval $T_{\ell}$. Similarly, (A.18) implies that $w_{\ell-1}\left(x_{\ell-1}(t)\right)>0, \forall t \in T_{\ell-1}$ and $\dot{x}_{\ell-1}(t)=0, \forall t \in T_{\ell}$. Then, $w_{\ell-1}\left(x_{\ell-1}(t)\right)>0, \forall t \in T_{\ell}$ and thus, using (3) and (A.15) in (A.24) yields

$$
\dot{h}_{\ell-1}(t)>0, \quad \forall t \in T_{\ell} .
$$

Therefore, combining (A.15), (A.20), (A.22), and (A.25) we conclude that the $j$ th switching function satisfies

$$
\begin{aligned}
& h_{j}(t)= \begin{cases}\alpha_{j}, & \forall 2 \leq j \leq \ell, \forall t \in \bigcup_{i=0}^{j-2} T_{i}, \\
-\frac{1}{E_{T}}, & \forall j \leq \ell, \forall t \in T_{j},\end{cases} \\
& \dot{h}_{j}(t)>0, \quad \forall j<\ell, \forall t \in T_{j+1}, \\
& \dot{h}_{j}(t)=0, \quad \forall j<\ell, \forall t \in \bigcup_{i=j+2}^{\ell} T_{i} .
\end{aligned}
$$

In order to clarify the idea, a schematic plot of the switching functions $h_{\ell-2}(t), h_{\ell-1}(t)$ and $h_{\ell}(t)$ is depicted in Fig. A.1.

The idea is then to show that the form of time courses in Fig. A.1 implies that the only enzyme that can be nonzero in $T_{\ell+1}$ is $u_{\ell+1}$ (as expressed in (A.19)). We proceed by analyzing the effect of enzyme $u_{j}$ being active in interval $T_{\ell+1}$.

- Case $j<\ell$ :

Assume that for some $j<\ell, u_{j} \in U_{\ell+1}^{*}$. Then in order to satisfy (A.15), (A.26)(A.28) imply that $h_{j}(t)$ must be discontinuous at $t=t_{\ell}$ (see Fig. A.1), which from (A.3) is not possible since both $\mathbf{x}(t)$ and $\mathbf{p}(t)$ are continuous. Hence, it follows that

$$
u_{j} \notin U_{\ell+1}^{*}, \quad \forall j<\ell .
$$

- Case $j>\ell+1$ :

Assume that for some $j>\ell+1, u_{j} \in U_{\ell+1}^{*}$. Then from (A.17) we have that $x_{i}\left(t_{\ell}\right)=0, \forall i>\ell+1$ and hence using (4) we conclude that $x_{i}(t)=0, \forall i>\ell+1$,

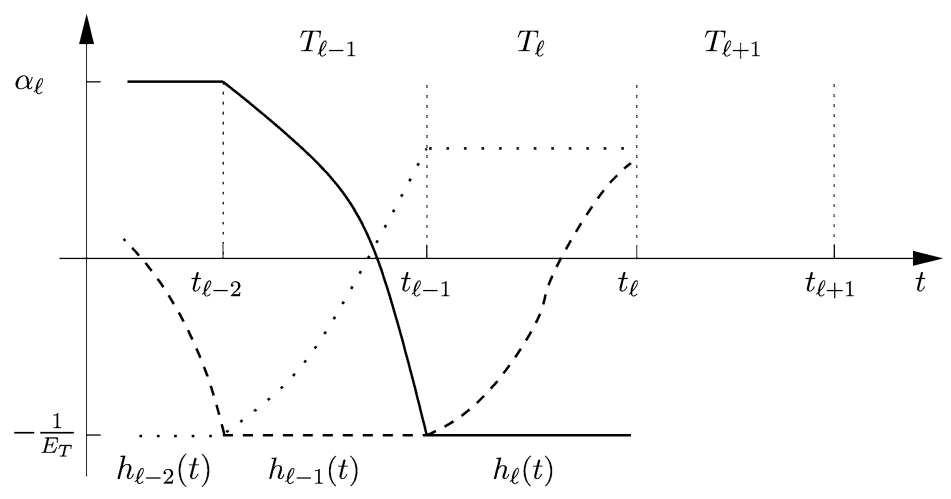

Fig. A.1 Sketch plot of switching functions $h_{\ell-2}(t), h_{\ell-1}(t)$ and $h_{\ell}(t)$. 
$\forall t \in T_{\ell+1}$. In view of (A.3) and (2), this implies that $h_{i}(t)=\alpha_{i} \geq 0, \forall i>\ell+1$, which contradicts (A.15). This means that $\mathbf{e}_{j}, j>\ell+1$, cannot be optimal in interval $T_{\ell+1}$ and thus we can use Fact 1 to conclude

$$
u_{j} \notin U_{\ell+1}^{*}, \quad \forall j>\ell+1 .
$$

- Case $j \in\{\ell, \ell+1\}$ :

Assume that $U_{\ell+1}^{*}=\{\ell, \ell+1\}$. Using (A.29) and (A.30) in (A.21) yields

$$
\begin{aligned}
& \dot{h}_{\ell}(t)=\left(p_{\ell+1}(t)-p_{\ell+2}\right) \frac{\partial w_{\ell+1}}{\partial x_{\ell+1}} w_{\ell}\left(x_{\ell}(t)\right) u_{\ell+1}(t), \quad \forall t \in T_{\ell+1}, \\
& \dot{h}_{\ell+1}(t)=-\left(p_{\ell+1}(t)-p_{\ell+2}\right) \frac{\partial w_{\ell+1}}{\partial x_{\ell+1}} w_{\ell}\left(x_{\ell}(t)\right) u_{\ell}(t), \quad \forall t \in T_{\ell+1} .
\end{aligned}
$$

Substituting (A.3) with $i=\ell+1$ in (A.31) and (A.32) leads to

$$
\begin{aligned}
& \dot{h}_{\ell}(t)=\left(\frac{\alpha_{\ell+1}-h_{\ell+1}(t)}{w_{\ell+1}\left(x_{\ell+1}(t)\right)}\right) \frac{\partial w_{\ell+1}}{\partial x_{\ell+1}} w_{\ell}\left(x_{\ell}(t)\right) u_{\ell+1}(t), \quad \forall t \in T_{\ell+1}, \\
& \dot{h}_{\ell+1}(t)=-\left(\frac{\alpha_{\ell+1}-h_{\ell+1}(t)}{w_{\ell+1}\left(x_{\ell+1}(t)\right)}\right) \frac{\partial w_{\ell+1}}{\partial x_{\ell+1}} w_{\ell}\left(x_{\ell}(t)\right) u_{\ell}(t), \quad \forall t \in T_{\ell+1} .
\end{aligned}
$$

Since $x_{\ell+1}(t) \neq 0, \forall t \in T_{\ell+1}$, (A.33) and (A.34) are well defined. From (A.14) and (A.15), $U_{\ell+1}^{*}=\{\ell, \ell+1\}$ implies that $\dot{h}_{\ell}(t)=\dot{h}_{\ell+1}(t)=0, \forall t \in T_{\ell+1}$, but in view of (A.33) and (A.34), this can only hold if $u_{\ell}(t)=u_{\ell+1}(t)=0, \forall t \in T_{\ell+1}$, which according to (A.11) is a contradiction. Moreover, if $U_{\ell+1}^{*}=\left\{u_{\ell}\right\}$, then $U_{\ell+1}^{*}=U_{\ell}^{*}$, contradicting the fact that $U_{i}^{*} \neq U_{i+1}^{*}, \forall i=0,1, \ldots, q-1$. Thus, $\mathbf{e}_{\ell}$ cannot be optimal in the interval $T_{\ell+1}$ and resorting to (A.16), the result (A.19) is obtained.

Finally, to conclude the argument, consider interval $T_{0}$ and assume that vertex $\mathbf{e}_{j}$, $j>0$, is optimal in $T_{0}$, then since $\mathbf{x}(0)=0$ it follows from (4) that $\mathbf{x}(t)=0, \forall t \in T_{0}$. From (2) and (A.3), this yields $h_{j}(t)=\alpha_{i} \geq 0, \forall i>0$, which contradicts (A.15), and therefore $\mathbf{e}_{j}, j>0$, cannot be optimal in interval $T_{0}$. Fact 1 then yields

$$
U_{0}^{*}=\left\{u_{0}\right\}
$$

We now consider interval $T_{1}$. If $\mathbf{e}_{j}, \forall j>1$, is optimal in interval $T_{1}$, then (A.35) implies $x_{i}\left(t_{0}\right)=0, \forall i>1$, and hence (4) yields $x_{i}(t)=0, \forall i>1, \forall t \in T_{1}$. From (2) and (A.3), this implies that $h_{i}(t)=\alpha_{i} \geq 0, \forall i>1$, which contradicts (A.15). Thus, from Fact 1 we conclude that $u_{j} \notin U_{1}^{*}, \forall j>1$. Now suppose that $\mathbf{e}_{0}$ and $\mathbf{e}_{1}$ are optimal in interval $T_{1}$, then similarly as in case $j \in\{\ell, \ell+1\}$ of Fact 2 (take (A.31)-(A.34) with $\ell=0)$, it can be shown that $\dot{h}_{0}(t)=\dot{h}_{1}(t)=0, \forall t \in T_{1}$ only when $u_{0}(t)=u_{1}(t)=0$, $\forall t \in T_{1}$, which in view of (A.11) is a contradiction. Moreover, if $\mathbf{e}_{0}$ is optimal in $T_{1}$, then $U_{1}^{*}=U_{0}^{*}$, contradicting our hypothesis that $U_{i}^{*} \neq U_{i+1}^{*}, \forall i=0,1, \ldots, q$. Thus, we conclude that $u_{0} \notin U_{1}^{*}$ and (A.16) yields

$$
U_{1}^{*}=\left\{u_{1}\right\}
$$


Eq. (A.35) and (A.36) imply that $x_{i}\left(t_{1}\right)=0, \forall i>1$, and therefore, we can inductively use Fact 2, leading to the desired result (A.12).

To prove (A.13), consider interval $T_{n}$, so that from (A.12) it holds that $U_{n}^{*}=\left\{u_{n}\right\}$. Then from (A.14) it follows that $h_{n}(t)=\min \left\{h_{0}(t), h_{1}(t), \ldots, h_{n}(t)\right\}, \forall t \in T_{n}$, but from (A.26)-(A.28) we have that it does not exist $h_{i}(t), i \neq n$ such that $h_{i}(t) \leq h_{n}(t)$ for $t \geq$ $t_{n-1}$, thus implying that $\dot{x}_{n}(t)<0, \forall t \geq t_{n-1}$. This in turn means that $\lim _{t \rightarrow \infty} x_{n}(t)=0$, and therefore the terminal condition (9) fails and $t_{f}$ grows without bound. This leads to the conclusion that $u_{n}(t)$ is zero during the whole optimization interval and (A.13) follows.

Open Access This article is distributed under the terms of the Creative Commons Attribution Noncommercial License which permits any noncommercial use, distribution, and reproduction in any medium, provided the original author(s) and source are credited.

\section{References}

Alves, R., Savageau, M.A., 2000. Extending the method of mathematically controlled comparison to include numerical comparisons. Bioinformatics 16(9), 786-798.

Banga, J., Balsa-Canto, E., Moles, C., Alonso, A., 2005. Dynamic optimization of bioprocesses: Efficient and robust numerical strategies. J. Biotechnol. 117, 407-419.

Bilu, Y., Shlomi, T., Barkai, N., Ruppin, E., 2006. Conservation of expression and sequence of metabolic genes is reflected by activity across metabolic states. PLoS Comput. Biol. 2(8), 932-938.

Brown, G., 1991. Total cell protein concentration as an evolutionary constraint on the metabolic control distribution in cells. J. Theor. Biol. 153, 195-203.

Campbell, N., 2004. Timing is everything. Nat. Rev. Genet. 5, 405.

Chaves, M., Albert, R., Sontag, E., 2005. Robustness and fragility of Boolean models for genetic regulatory network. J. Theor. Biol. 235, 431-449.

Cornish-Bowden, A., 2004a. Fundamentals of Enzyme Kinetics, 3rd edn. Portland Press.

Cornish-Bowden, A., 2004b. The Pursuit of Perfection: Aspects of Biochemical Evolution. Oxford University Press, USA.

Heinrich, R., Klipp, E., 1996. Control analysis of unbranched enzymatic chains in states of maximal activity. J. Theor. Biol. 182, 242-252.

Heinrich, R., Schuster, S., 1996. The Regulation of Cellular Systems. Chapman \& Hall, London.

Heinrich, R., Schuster, S., Holzhütter, H.G., 1991. Mathematical analysis of enzymatic reaction systems using optimization principles. Eur. J. Biochem. 201, 1-21.

Holzhütter, H.-G., 2004. The principle of flux minimization and its application to estimate stationary fluxes in metabolic networks. Eur. J. Biochem. 271, 2905-2922.

Ibarra, R., Edwards, J., Palsson, B., 2002. Escherichia coli K-12 undergoes adaptive evolution to achieve in silico predicted optimal growth. Nature 420, 186-189.

Kalir, S., McClure, J., Pabbaraju, K., Southward, C., Ronen, M., Leibler, S., Surette, M.G., Alon, U., 2001. Ordering genes in a flagella pathway by analysis of expression kinetics from living bacteria. Science 292, 2080-2083.

Klipp, E., Heinrich, R., 1999. Competition for enzymes in metabolic pathways: Implications for optimal distributions of enzyme concentrations and for the distribution of flux control. Biosystems 54, 1-14.

Klipp, E., Heinrich, R., Holzhütter, H.G., 2002. Prediction of temporal gene expression: Metabolic optimization by re-distribution of enzyme activities. Eur. J. Biochem. 269, 5406-5413.

Klipp, E., Herwig, R., Kowald, A., Wierling, C., Lehrach, H., 2005. Systems Biology in Practice: Concepts, Implementation and Application. Wiley-Vch, New York.

Leng, X., Müller, H.-G., 2006. Time ordering of gene coexpression. Biostatistics 7(4), 569-584.

Llorens, M., Nuno, J., Rodriguez, Y., Melendez-Hevia, E., Montero, F., 1999. Generalization of the theory of transition times in metabolic pathways: A geometrical approach. Biophys. J. 77, 22-36.

Mahadevan, R., Edwards, J., Doyle, F., 2002. Dynamic flux balance analysis of diauxic growth in Escherichia coli. Biophys. J. 83, 1331-1340.

Meléndez-Hevia, E., Torres, N., Sicilia, J., 1990. A generalization of metabolic control analysis to conditions of no proportionality between activity and concentrations of enzymes. J. Theor. Biol. 142, 443-451. 
Nielsen, J., 2007. Principles of optimal network operation. Mol. Syst. Biol. 3(126), 00-00.

Ou, J., Yamada, T., Nagahis, K., Hirasawa, T., Furusawa, C., Yomo, T., Shimizu, H., 2008. Dynamic change in promoter activation during lysine biosynthesis in Escherichia coli cells. Mol. Biosyst. 4, 128-134.

Oyarzún, D., Ingalls, B., Kalamatianos, D., 2007. Optimal metabolic regulation by time varying enzyme activities: A control theoretic approach. In: Proceedings of Foundations of Systems Biology \& Engineering, Stuttgart, Germany

Pontryagin, L.S., Boltyanskii, V., Gamkrelidze, R., Mischenko, F., 1962. The Mathematical Theory of Optimal Processes. New York, Wiley (transl. by K.M. Tririgoff).

Rutquist, P., Edvall, M., (2009) PROPT-Matlab Optimal Control Software, Tomlab Optimization Inc., 260 SE Bishop Blvd Ste E, Pullman, WA 99163, USA, February 2009

Savageau, M.A., 1974. Optimal design of feedback control by inhibition: steady state considerations. J. Mol. Evol. 4, 139-156.

Savageau, M.A., 1975. Optimal design of feedback control by inhibition: Dynamic considerations. J. Mol. Evol. 5(3), 199-222.

Savageau, M.A., 1976. Biochemical Systems Analysis: a Study of Function and Design in Molecular Biology. Addison-Wesley, Reading.

Savageau, M.A., 1985. A theory of alternative designs for biochemical control systems. Biomed. Biochim. Acta 44(6), 875-880.

Schuetz, R., Kuepfer, L., Sauer, U., 2007. Systematic evaluation of objective functions for predicting intracellular fluxes in Escherichia coli. Mol. Syst. Biol. 3, 119.

Schuster, S., Pfeiffer, T., Fell, D., 2008. Is maximization of molar yield in metabolic networks favoured by evolution? J. Theor. Biol. 252(3), 497-504.

Torres, N., 1994. Application of the transition time of metabolic systems as a criterion for optimization of metabolic processes. Biotechnol. Bioeng. 44, 291-296.

Torres, N., Voit, E., 2002. Pathway Analysis and Optimization in Metabolic Engineering. Cambridge University Press, Cambridge.

Uygun, K., Matthew, H., Huang, Y., 2006. DFBA-LQR: An optimal control approach to flux balance analysis. Ind. Eng. Chem. Res. 45, 8554-8564.

van Riel, N., Giuseppin, M., Verrips, C., 2000. Dynamic optimal control of homeostasis: An integrative systems approach for modeling of the central nitrogen metabolism in Saccharomyces cerevisiae. Metab. Eng. 2, 14-33.

Varma, A., Palsson, B., 1994. Metabolic flux balancing-basic concepts, scientific and practical use. BioTechnology 12(10), 994-998.

Varner, J., Ramkrishna, D., 1999. Metabolic engineering from a cybernetic perspective. 1. Theoretical preliminaries. Biotechnol. Prog. 15, 407-425.

Vera, J., de Atauri, P., Cascante, M., Torres, N., 2003. Multicriteria optimization of biochemical systems by linear programming: Application to production of ethanol by Saccharomyces cerevisiae. Biotechnol. Bioeng. 83, 335-343.

Zaslaver, A., Mayo, A., Rosenberg, R., Bashkin, P., Sberro, H., Tsalyuk, M., Surette, M., Alon, U., 2004. Just-in-time transcription program in metabolic pathways. Nat. Genet. 36(5), 486-491. 